\title{
Force-Velocity Modulation Strategies for Soft Tissue Examination
}

\author{
Jelizaveta Konstantinova, Min Li, Vahid Aminzadeh, Prokar Dasgupta, Kaspar Althoefer, Member, \\ IEEE, and Thrishantha Nanayakkara, Member, IEEE
}

\begin{abstract}
Advanced tactile tools in minimally invasive surgery have become a pressing need in order to reduce time and improve accuracy in localizing potential tissue abnormalities. In this regard, one of the main challenges is to be able to estimate tissue parameters in real time. In palpation, tactile information felt at a given location is identified by the viscoelastic dynamics of the neighboring tissue. Due to this reason the tissue examination behavior and the distribution of viscoelastic parameters in tissue should be considered in conjunction. This paper investigates the salient features of palpation behavior on soft tissue determining the effectiveness of localizing hard nodules. Experimental studies involving human participants, and validation tests using finite element simulations and a tele-manipulator, were carried out. Two distinctive tissue examination strategies in force-velocity modulation for the given properties of target tissue were found. Experimental results suggest that force-velocity modulations during continuous path measurements are playing an important role in the process of mechanical soft tissue examination. These behavioral insights, validated by detailed numerical models and robotic experimentations shed light on future designs of optimal robotic palpation.
\end{abstract}

\section{INTRODUCTION}

The technological development in the field of surgical robotics made it possible to enhance the quality of various surgical procedures, robot-assisted Minimally Invasive Surgery (MIS), for instance. This procedure is performed using tele-manipulator robotic devices which move through small incisions in a patient body. Shorter recovery times and reduced traumatism for patients are among various benefits of such procedures[1]. MIS requires the use of miniature and dexterous surgical tools, which continually evolve [2], [3]. However, there still exists a need to develop more advanced surgical tools, such as tactile probes for intra-operative soft tissue examination and tumor detection. Currently, tactile feedback is completely disregarded in robotic MIS and the navigation of tools during surgery is performed remotely with the help of three-dimensional visual displays. The implementation of tactile probing devices can help to provide tactile feedback information to the surgeon and to improve clinical outcomes [4].

\footnotetext{
* This work was supported by STIFF-FLOP project grant from the European Commission Seventh Framework Programme under agreement 287728 and National Institute for Health Research (NIHR) Biomedical Research Centre based at Guy's and St Thomas' NHS Foundation Trust and King's College London.

J. Konstantinova, M. Li, V. Aminzadeh, K. Althoefer and T. Nanayakkara are with the Department of Informatics, King's College London, Strand, London WC2R 2LS, U.K. (e-mail: \{jelizaveta.zirjakova, min.m.li, vahid.aminzadeh, kaspar.althoefer, thrish.antha \}@kcl.ac.uk).

P. Dasgupta is with the MRC Centre for Transplantation, DTIMB and NIHR BRC, King's College London, Guy's Hospital, London SE1 9RT, U.K. (e-mail: prokarurol@gmail.com).
}

The area of tactile sensing for MIS has been extensively studied lately. There are various promising projects of tactile devices in this field, which, however, are not yet used for real medical applications, described in [5-8]. This is mainly because only tested and certified devices can be used in surgery. The results of artificial tactile examination, while organ probing or palpation, should be accurate, robust and efficient [9]. Various factors, such as nonlinear mechanical soft tissue properties, mobility of organs, blood flows and patient breathing can influence the correct results of tactile data, thus providing large variability over several trials [10], [11]. Therefore, any soft tissue examination probe for MIS should be able to take into account the influence of the environment and work under variable conditions with an optimal behavior.

To solve this problem, the knowledge about various tissue examination strategies should be developed. It can enhance the quality of soft tissue tactile information and improve the accuracy in abnormality detection during tactile probing. Hereby, one needs to study how humans palpate soft tissue, as palpation is used during open surgery for diagnostic purposes. Surgeons rely on tactile information to assess the location, structure and shape of a potential tumor. But the strategies and behaviors of manual palpation are not standardized and can vary depending on the clinician's preferences [12]. Moreover, there exist various techniques used for the examination of specific organs. For instance, during clinical breast palpation, usually, vertical or spiral patterns are applied to scan the whole surface of the breast and to locate sites of potential abnormalities. Then circular movements with variable pressure or tissue rolling between fingers is applied to scan suspicious areas [13]. Finger tapping, vibration and sliding are popular techniques to check the presence of abnormality during prostate palpation [14]. Usually, various techniques are combined during one examination to enhance the general result [15]. Moreover, the efficiency of palpation largely depends on experience of a person and physical capabilities of human tactile perception [16].

There are various clinically related works, where palpation techniques for different body parts and organs are evaluated and compared [17], [18]. Such approaches discuss the performance of each technique for abnormality detection and develop sets of recommendations to increase efficiency of palpation. However, the physical quantification of each technique and its impact on abnormality localization is not discussed. Work in [19] has evaluated the impact of force and velocity during virtual palpation of simulated materials with different stiffness values. The results have shown the dependence of these two factors on the properties of the environment. The focus of this paper is to study manual palpation of soft media with hard inclusions to understand the 
impact of the main behavioral features of this procedure, such as applied force and finger velocity. Strategies used to locate an abnormality are studied for prospective implementation of this knowledge in artificial probing systems that could be used during MIS.

In Section II, information about palpation studies is presented; Section III presents the mathematical modeling of the obtained findings. The probing strategies are implemented and evaluated using a tele-manipulator setup with tactile sensing probe in Section IV. In Section V the results and discussion are presented.

\section{PALPATION STUDIES}

\section{A. Experimental Setup}

\section{1) Experimental Protocol and Subjects}

The target of the palpation studies was to understand the mechanisms employed by humans to detect harder formations in soft media and to evaluate exerted finger pressure and palpation velocities. The experimental protocol of this work was approved by the King's College London Biomedical Sciences, Dentistry, Medicine and Natural \& Mathematical Sciences Research Ethics Subcommittee. Palpation tests, carried out in the framework of this work, were implemented involving sixteen human participants eight experts with medical background and at least five years experience in manual palpation, and eight novices with regards to medical training. The subjects were asked to palpate the phantom tissue in a linear unidirectional way in order to sense hard inclusions over the defined measurement path. Each test consisted of five trials.

Three hard nodules, placed $30 \mathrm{~mm}$ apart with a diameter of $10 \mathrm{~mm}$, were embedded in the silicone phantom $\left(100 \times 100 \times 30 \mathrm{~mm}^{3}\right)$ at different depths from the surface -2 $\mathrm{mm}, 6 \mathrm{~mm}$ and $11 \mathrm{~mm}$. To fabricate the soft tissue phantom, silicone gel compound RTV6166 (Techsil Limited, UK) with ratio 4:6 and stiffness of $900 \mathrm{mPa} \cdot \mathrm{s}$ was used. Hard silicone compound RTV615 (Techsil Limited, UK), ratio 10:1 and viscosity $4000 \mathrm{mPa} \cdot \mathrm{s}$, was employed for the nodule fabrication. Thus, the relation of stiffness between soft tissue and abnormity is kept, as the abnormality is typically stiffer [20].

\section{2) Force Measurements}

To evaluate finger velocity and pressure of the subjects during manual palpation, an experimental setup was developed, Fig. 1. The force and torque values, applied by the finger, were measured with an industrial force / torque sensor Mini 40 (ATI industrial automation), which was placed under a transparent supporting plate for silicone phantom. It has a sensing capability of $6 \mathrm{DOF}$ and a force sensing range of $\pm 10 \mathrm{~N}$ in lateral directions and $\pm 30 \mathrm{~N}$ in normal direction. The resolution of normal force is $0.01 \mathrm{~N}$ allowing acquiring accurate data.

\section{3) Movement Tracking}

A three-dimensional vision system (Microsoft Kinect camera) is used to record finger movements during palpation. The Kinect is equipped with a camera with $640 \times 480$ pixel resolution and a depth sensor, and has data acquisition frequency of $30 \mathrm{fps}$. To track movement of a subject's hand during palpation, the Microsoft Visual $\mathrm{C}++$ with OpenCV package was used. Thereby, three-dimensional position was recorded and used to calculate velocity. The accuracy of position detection for the camera depends on the speed of movement of an object. Evaluation tests, involving comparison with an alternative method of position tracking, were carried out to quantify the accuracy of the Kinect camera. The finger trajectories were recorded with a Kinect hand-tracking algorithm and two cameras, placed at a fixed distance. The stereovision display of the fingers equipped with colored marker was processed in MATLAB to obtain the trajectories. The result of the comparison of the two trajectories has shown that the position accuracy for the Kinect camera during the palpation movement tracking is 1-2 $\mathrm{mm}$, which is suitable for our studies.

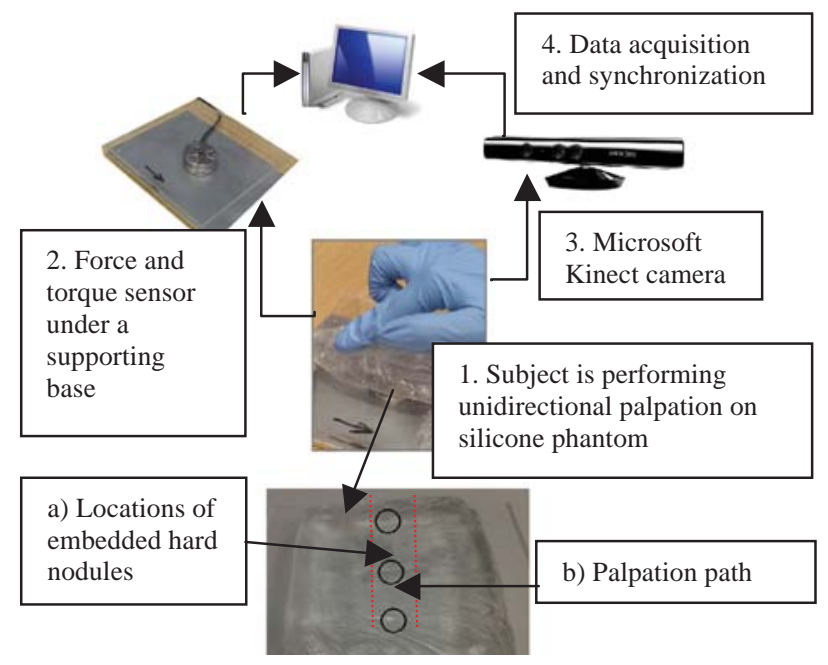

Fig. 1. The arrangement of the experimental setup: 1 - a subject is performing palpation of phantom tissue with three embedded nodules (a) in a unidirectional way on a predefined path of palpation (b); 2 - force and torque sensor measures load applied during palpation; 3 - Microsoft Kinect camera, using OpenCv algorithm, the hand trajectory; 4 - all data is recorded and synchronized

\section{B. Palpation Results}

The effects of palpation force and velocity need to be evaluated in the spatial context of hard nodule detection; therefore, one needs to analyze only those measurements in the neighborhood of the detected nodule. Five subjects detected the first nodule (embedded at $11 \mathrm{~mm}$ from the surface), and ten participants sensed the second nodule (located $6 \mathrm{~mm}$ deep from the surface).All sixteen subjects detected the third nodule located just $2 \mathrm{~mm}$ from the surface; no false positives occurred. Therefore, data obtained in the region of the third nodule was taken for the following analysis.

To analyze the gradual modulations of palpation force and velocity around the third nodule, this area was separated into several regions - bars, as shown on (Fig. 2). The width of the bars was set to $5 \mathrm{~mm}$ (radius of the nodule). There was no significant variance of force and velocity (average $5 \%$ ) inside each bar, according to experimental verification. Firstly, to understand the general pattern of force and velocity modulation for the region around the third nodule, mean values of applied force and velocity for six bars for each subject are shown in Fig.3 and Fig.4. Some distinctive trends 
can be observed for in the modulation of these two variables across the region of interest. Force and velocity tend to increase or decrease during the detection of a hard nodule. Therefore, the effect of each variable on the particular location in the area around the nodule should be tested.

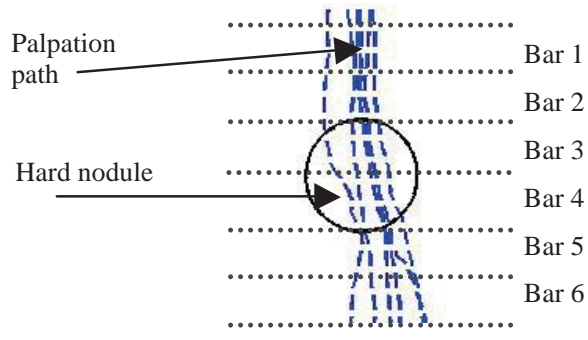

Direction of movement

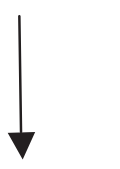

Fig. 2. Interval bars around the nodule (palpation path marked blue)

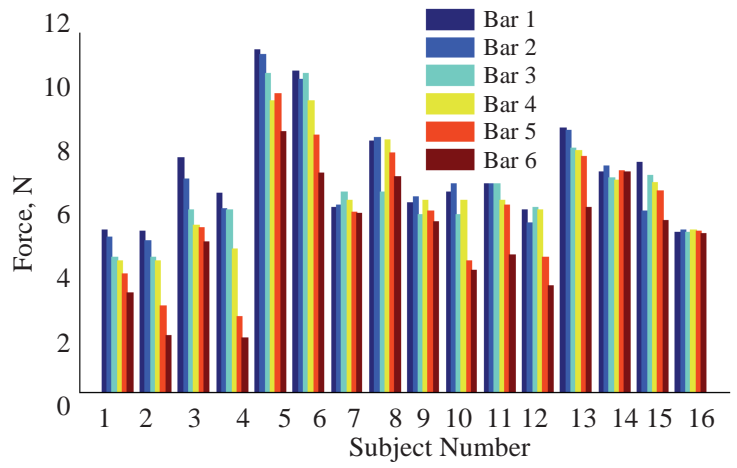

Fig.3. Force distribution across all subjects

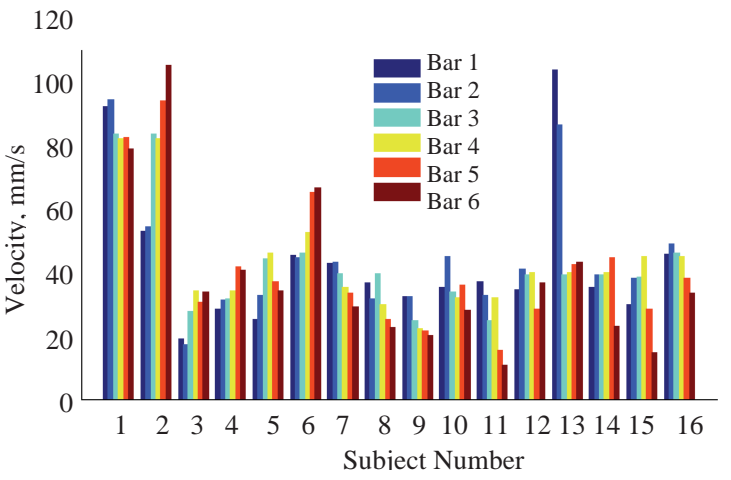

Fig.4. Velocity distribution across all subjects

A three-way analysis of variance (ANOVA) test was conducted to observe the significance of the variability of each factor - force, velocity, and individual subject - at various distances (Bars) from the nodule. The variation of applied force has a significant effect $\left(\mathrm{F}_{(3.96)}=14.9, \quad p<\right.$ $0.00001)$ on the location around the nodule. However there was no effect from velocity $\left(\mathrm{F}_{(3.96)}=2.1, p=0.15\right)$ and no effect from the impact of each individual subject $\left(\mathrm{F}_{(3.96)}=\right.$ $0.21, p=0.65)$. The separate analysis of the two groups of subjects, experts and novices, has shown the same result. Therefore, one can conclude that the modulation of finger pressure around the location of a hard inclusion is following a certain pattern, while the velocity modulation seems not to be following a specific pattern among subjects.

To understand the modulation of velocity and force around the nodule better, the inclination of the normalised magnitude of the variable should be studied. Therefore, a first-degree polynomial was fitted to the mean values of the variables for each Bar. Fig. 5confirms the statistical significance of force across all subjects and demonstrates the consistent decay of this variable. The velocity trends can only be observed in case the fitted line is displayed on two separate plots, as shown in Fig.6. One can observe that there are two behaviors of velocity strategy - it can either decrease or increase. In order to confirm the presence of two strategies in palpation over a hard nodule, slope (gradient) values of fitted lines of velocity $m(V)$ were plotted versus slope values of fitted lines of force $m(F)$ (Fig.7).Two trends of palpation behavior partitioned with $\mathrm{k}$-means clustering algorithm can be seen - decreasing (63\% of subjects) and increasing (37\% of subjects) velocity is accompanied with a decrease of force. Here it is worth to mention that experts are represented in both groups.

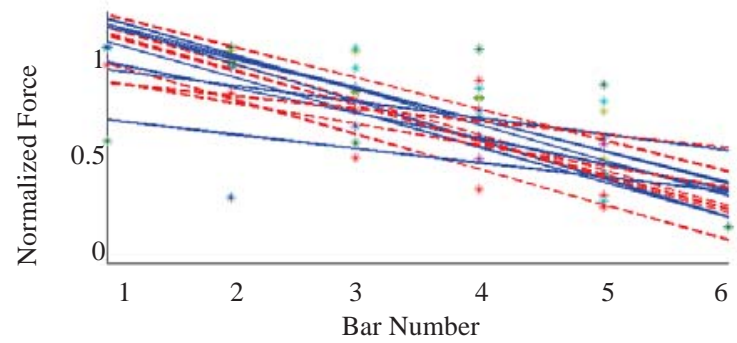

Fig. 5. Distribution of force for each subject shown with data points for each bar, fitted lines show inclination of force (dotted red line - experts, solid blue line - novices)

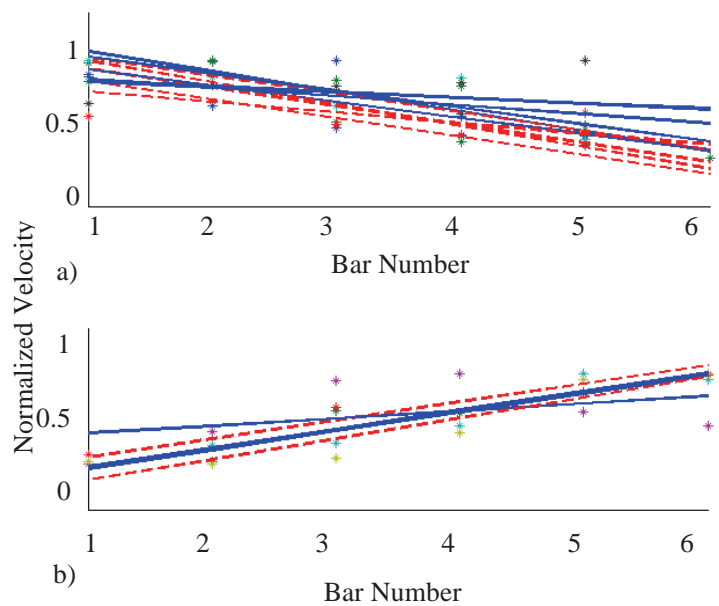

Fig.6. Distribution of velocity for each subject shown with data points for each bar a) first group of subject fitted lines show inclination of decreasing velocity, and b) for second group, fitted lines show inclination of increasing velocity:(dotted red line - experts, solid blue line - novices)

To check the statistical significance for both behaviors, a three-way ANOVA test was conducted again separately for the two groups of subjects with different palpation strategies. The result of the analysis for the strategy with decreasing velocity gradient has shown, the significant effect of both force $\left(\mathrm{F}_{(4.00)}=11.34, p<0.00001\right)$ and velocity magnitude $\left(\mathrm{F}_{(4.00)}=7.22, \quad p<0.00001\right)$ on the variability of data at various distances (Bars) from the nodule. Similarly, for the strategy with increasing velocity, these two factors are significant as well $\left(\left(\mathrm{F}_{(4.18)}=10.85, p<0.0001\right)\right.$ for force and 
$\left(\mathrm{F}_{(4.18)}=9.4, p<0.0001\right)$ for velocity magnitude $)$. Thus, our studies of palpation behavior have shown the presence of two strategies. The force-velocity modulation is used in the region of hard formation to detect the presence of an inclusion in two ways: a) the magnitude of applied velocity and force decreases, and b) the magnitude of applied velocity increases but the magnitude of force decreases.

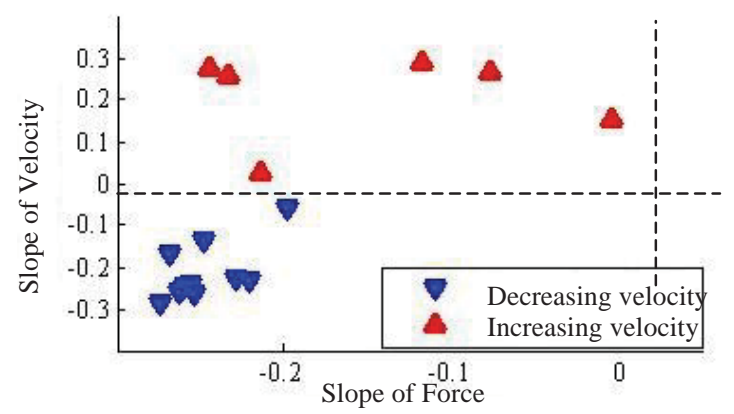

Fig.7. Slope values of fitted lines of force $m(F)$ plotted versus Slope values of fitted lines of velocity $m(V)$. Two clusters, obtained with k-means method are shown with blue and red dots

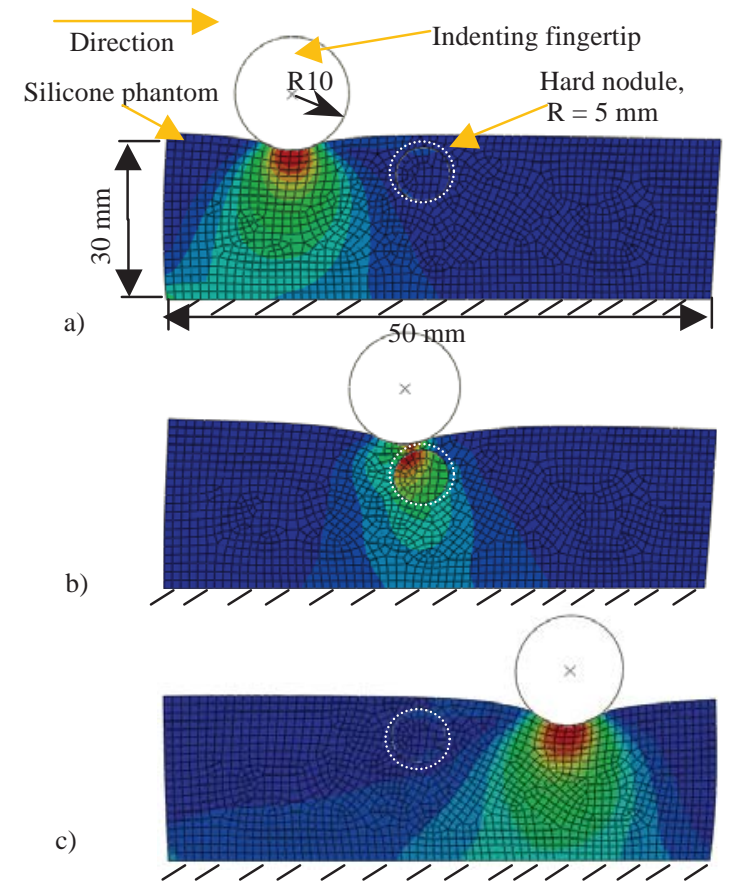

Fig. 8. Finite element simulation of silicone phantom indented with a fingertip (diameter $20 \mathrm{~mm}$ ) above the nodule location (diameter $10 \mathrm{~mm}$ ): a) initial position, b) location above the nodule, c) final position.

\section{INTERPRETATION OF PALPATION STRATEGIES}

\section{A. Finite Element Model of Silicone Phantom}

In this section the palpation strategies defined previously are modeled using finite element (FE) simulations in ABAQUS 6.10 software. The aim of this analysis is to understand the response of soft tissue during each strategy of palpation. In order to model the silicone phantom, the material properties are defined using non-linear, isotropic, incompressible, and hyperelastic Arruda-Boyce model, using constitutive equation obtained for the same silicone in [21]. The phantom tissue was modeled as a $50 \times 30 \mathrm{~mm}^{2}$ planar block, as shown in Fig. 8. The size of the element mesh was set as $1 \mathrm{~mm}$ using a four-node bilinear stress quadrilateral, reduced integration, hourglass control (CPS4R) element type. The diameter of the nodule was set to $10 \mathrm{~mm}$ and it was embedded $2 \mathrm{~mm}$ deep from the surface. To simulate a finger during manual palpation the discretely rigid spherical body was simulated as a mass of $0.1 \mathrm{~kg}$. The diameter of the sphere was set to $20 \mathrm{~mm}$, taking into account the limits of a fingertip contact area. The contact between simulated tissue and indenting body was set as frictionless, assuming perfect lubrication. The sphere was indented in the material at $3 \mathrm{~mm}$ for the first step of FE simulation. During the rest of simulation, the indentation depth was not controlled and it was moved along the normal direction of the simulated tissue.

\section{B. Simulated Tissue Responses for Palpation Strategies}

Modulations of palpation force and velocity around the location of a hard nodule were shown in Section II. The aim of FE simulations is to understand the stress responses from soft tissue during examination. The contact area with the simulated material is constant, thus, in this case, the stress can be directly compared with force. The obtained magnitude of force and velocity slopes, for both strategies, was applied on the simulated model of a phantom tissue with a hard nodule. To simulate the change of force applied by the subject, the weight of the simulated finger was varied. Four different examination strategies were tested: a) increased velocity and decreased force; b) decreased velocity and decreased force; c) constant velocity and decreased force; d) constant velocity and constant force. Fig.9 displays the stress magnitude of the indentation contact point along the movement path. It can be observed that the maximum stress distribution for all types of exposure is achieved as the nodule is touched. The stress distribution is more or less uniform for the decreased force and velocity magnitude (b). The exposure with increased velocity and decreased force results to high stress feedback before the location of a nodule (a). While stress response for the strategies (c and d) with constant velocity contains some peaks after the nodule is detected.

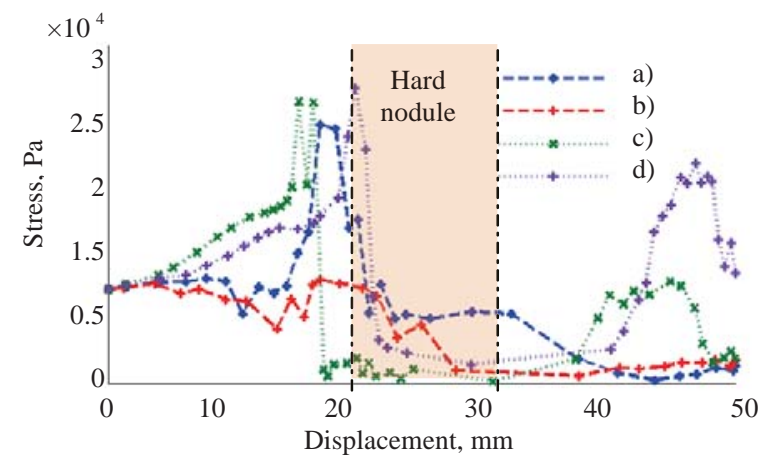

Fig.9. Stress component over palpation path for a) increased velocity and decreased load; b) decreased velocity and decreased load; c) constant velocity and decreased load; d) constant velocity and constant load

The results of FE simulations have shown that force velocity modulations applied during the palpation of a hard nodule influence the stress distribution in soft tissue. 
Therefore, these palpation parameters should be taken into account during soft tissue examination.

\section{VAlidation OF PALPATION StRATEgIES}

In order to understand and interpret palpation strategies and to validate simulation results, it is important to study tactile feedback sensed by participants. This section describes experiments carried out on the tele-manipulation setup [22].To study how the strategies, observed in the Section II, are sensed by t he human, a tele-manipulator setup was used to scan the silicone phantom with the help of a tactile probe in two ways:(a) with decreasing and (b) with increasing velocity. Similarly to the palpation studies, an examination was performed in a linear unidirectional way over the location of the hard nodule. Ten experiments were carried out for each strategy to measure the force feedback sensed by a tactile probe of the silicone phantom.

\section{A. Tele-manipulator Setup}

Fig.10 shows the arrangement of the setup. A user implements the position control of the robot arm (Fanuc M$6 \mathrm{iB})$ with the help of haptic device (PHANTOM Omni, Sensable). The average delay of the system is $0.25 \pm 0.04 \mathrm{~s}$ and the average position error $0.48 \mathrm{~mm}$. The resultant position of the robot end effector is displayed in the virtual environment, which is the virtual model of the phantom tissue. A sliding tactile probe with a spherical indenter $(6 \mathrm{~mm}$ diameter), attached to the end effector of the robot, was used to measure force feedback from the phantom tissue. A force / torque 6DOF sensor Nano17 (ATI industrial automation, force resolution $1 / 320 \mathrm{~N}$ ) was employed as a sensing element integrated at the tip of the probe. In order to reduce the effect of friction the surface of the phantom was lubricated. Stiffness values were calculated using force data and indentation depth of the probe, and displayed on the screen as a colormap. In addition, a camera was used to observe the position of the probe during phantom examination in real time.

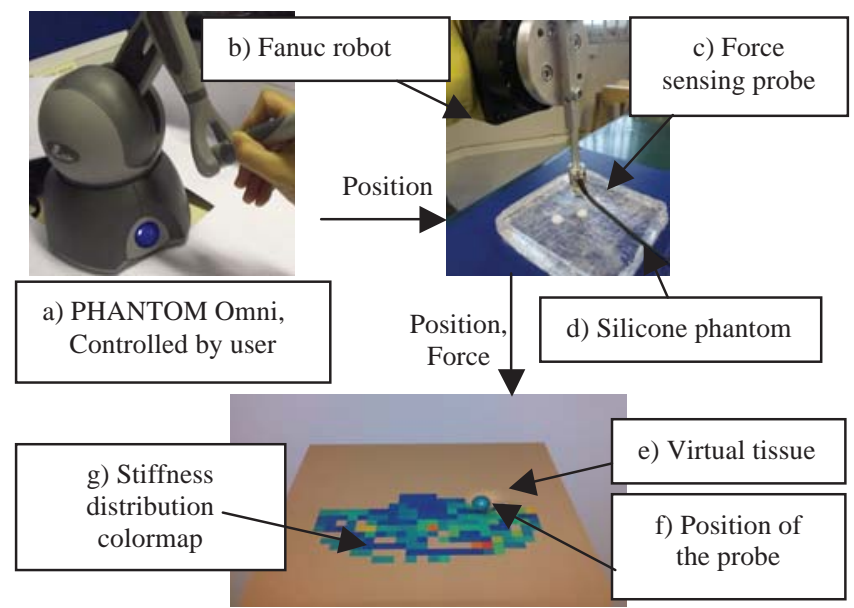

Fig.10. Tele-manipulator setup: a) Haptic device PHANTOM Omni (master) is used to control the position; b) Fanuc robot (slave) is following the trajectory; c) Force sensing probe is scanning the silicone phantom (d); e) Virtual tissue is displayed on the computer monitor; f) Position of the probe is displayed in real time; g) Stiffness distribution colormap is generated using force and indentation depth data

\section{B. Results of Tele-manipulation}

The results of forces recorded during tele-manipulation (path over the nodule, ten trails) are shown in Fig. 11 and Fig.12. As expected, the feedback force profiles are different for each probing strategy and can provide information about the palpation behavior, applied during each strategy.

If the indentation depth of the probe is constant during examination, the areas of high stiffness (hard nodule) are denoted with increased force feedback. But the indentation depth is not controlled for this experiment. Thus force feedback for decreasing velocity (Fig. 11) has two peaks and a steep trough around the location of the nodule. This means the indentation depth above the nodule has reached a minimum. In other words, the probe has followed the shape of the nodule, while subjects were relying on the kinesthetic sensing - the location of the finger in space during palpation. This observation corresponds to the simulation shown in Fig.9 b), as stress magnitude did not vary significantly around the location of the nodule. Assuming constant contact area, a subject senses higher feedback force as the stress of that area is higher. That allows comparing force feedback detected by the tactile probe and stress magnitude from FE simulations.

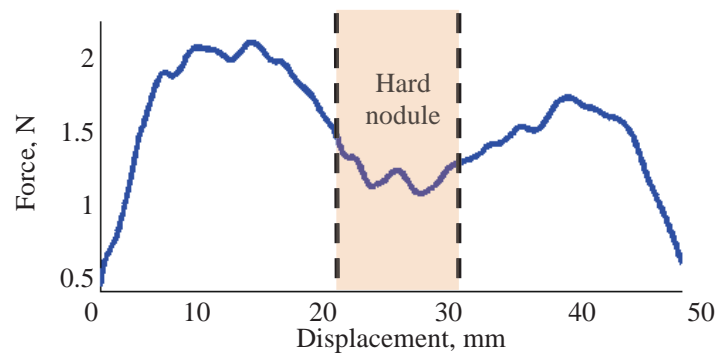

Fig. 11. Force feedback sensed with probe based on decreasing velocity strategy

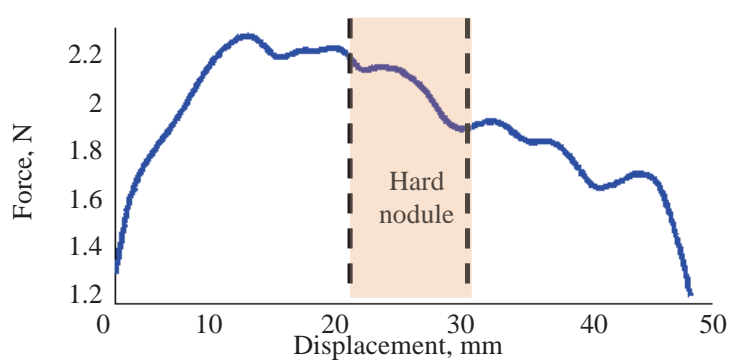

Fig.12. Force feedback sensed with probe based on increasing velocity strategy

The force profile, measured by the probe, for the increasing velocity strategy (Fig.12) gradually decreases within the marked area. First, the peak is reached just before the location of the nodule. The force drops along with the decrease of the velocity, i.e. when an inclusion is sensed. As discussed, the force feedback can be the determining factor during hard area localization if indentation is kept constant. Thus, as opposed to the first strategy, the most likely is that the received force feedback, in this case, is the determining factor for the localization of the hard nodule. The drop of the force can be explained by the fact that the location of the nodule is already detected. The simulation in Fig.9a) supports 
the results obtained during tele-manipulation - the stress magnitude has reached its peak before the nodule.

\section{CONCLUSIONS}

This paper provides an analysis of palpation behavior and presents the strategies for a mechanical examination of soft tissue. Our findings clearly show two distinct strategies that are applied during finger-based palpation of soft materials that contain embedded hard inclusions. For each strategy, FE simulations and validation experiments on the telemanipulation setup were carried to support the experimental results.

The results show how the interaction between a finger and a soft tissue influences the way a hard nodule is detected. The correct combination of all variables (probing force and velocity) significantly improves the tumor localization process. The state variables of the palpation strategies are summarized in Table I.

\section{TABLE I. PALPATION STRATEGIES}

\begin{tabular}{|l|l|l|l|}
\hline \multirow{2}{*}{ Strategy } & \multicolumn{3}{|c|}{ State variables with respect to the nodule location } \\
\cline { 2 - 4 } & Velocity & Pressure applied & $\begin{array}{c}\text { Most likely } \\
\text { determining factor }\end{array}$ \\
\hline 1 & Decreasing & Decreasing & $\begin{array}{l}\text { Kinesthetic feedback/ } \\
\text { Displacement }\end{array}$ \\
\hline 2 & Increasing & Decreasing & Force feedback \\
\hline
\end{tabular}

It was shown, that the different determining factors are being used during each strategy to detect hard inclusions. Thus, the detection and localization of malignant and benign formations can be significantly improved by using one of the palpation strategies or by alternating between the two of them. This implies that the reliability of detecting abnormal tissue sites depends not only on the sensitivity of the probe itself, but also the force/velocity control strategy too. This arises from the fact that the interaction dynamics between the probe and the tissue depends on how the applied force and probing velocity interact with the viscoelastic dynamics of a non-homogeneous tissue. The unpublished extension of this work reveals the significant impact of the type of soft tissue on the palpation results. We believe that the findings can provide valuable design guidelines for new probing devices, which would measure soft tissue properties along a continuous probing path.

\section{ACKNOWLEDGMENT}

The research has received funding from the European Commission's Seventh Framework Programme, project STIFF-FLOP (Grant No: 287728) and the National Institute for Health Research (NIHR) Biomedical Research Centre based at Guy's and St Thomas' NHS Foundation Trust and King's College London. The views expressed are those of the authors and not necessarily those of the NHS, the NIHR or the Department of Health.

\section{REFERENCES}

[1] D. B. Camarillo, T. M. Krummel, and J. K. Salisbury, "Robotic technology in surgery: past, present, and future.," American journal of surgery, vol. 188, no. 4A Suppl, p. 2S-15S, Oct. 2004.

[2] M. Eltaib, "Tactile sensing technology for minimal access surgery-a review," Mechatronics, vol. 13, no. 10, pp. 1163-1177, Dec. 2003.
[3] P. Puangmali, K. Althoefer, L. D. Seneviratne, D. Murphy, and P. Dasgupta, "State-of-the-Art in Force and Tactile Sensing for Minimally Invasive Surgery," IEEE Sensors Journal, vol. 8, no. 4, pp. 371-381, Apr. 2008.

[4] A. G. Harrell and B. T. Heniford, "Minimally invasive abdominal surgery: lux et veritas past, present, and future.," American journal of surgery, vol. 190, no. 2, pp. 239-243, 2005.

[5] H. Liu, D. P. Noonan, B. J. Challacombe, P. Dasgupta, L. D. Seneviratne, and K. Althoefer, "Rolling mechanical imaging for tissue abnormality localization during minimally invasive surgery.," IEEE transactions on bio-medical engineering, vol. 57, no. 2, pp. 404-14, Feb. 2010.

[6] Y. Tanaka, Q. Yu, K. Doumoto, A. Sano, Y. Hayashi, M. Fujii, Y. Kajita, M. Mizuno, T. Wakabayashi, and H. Fujimoto, "Development of a real-time tactile sensing system for brain tumor diagnosis.," International journal of computer assisted radiology and surgery, vol. 5, no. 4, pp. 359-67, Jul. 2010.

[7] Y. Hu, R. B. Katragadda, H. Tu, Q. Zheng, Y. Li, and Y. Xu, "Bioinspired 3-D Tactile Sensor for Minimally Invasive Surgery," Journal of Microelectromechanical Systems, vol. 19, no. 6, pp. 14001408, Dec. 2010.

[8] R. Ahmadi, M. Packirisamy, and J. Dargahi, "Optical Fiber Sensor Array for Artery / Lump Detection," Industrial Engineering, no. 1, pp. $1-4,2011$.

[9] G. Tholey, J. P. Desai, and A. E. Castellanos, "Force Feedback Plays a Significant Role in Minimally," Annals of Surgery, vol. 241, no. 1, pp. 102-109, 2005.

[10] S. Schostek, M. O. Schurr, and G. F. Buess, "Review on aspects of artificial tactile feedback in laparoscopic surgery.," Medical engineering \& physics, vol. 31, no. 8, pp. 887-98, Oct. 2009.

[11] P. Puangmali, H. Liu, L. D. Seneviratne, P. Dasgupta, and K. Althoefer, "Miniature 3-Axis Distal Force Sensor for Minimally Invasive Surgical Palpation," IEEE/ASME Transactions on Mechatronics, vol. 17, no. 4, pp. 646-656, 2011.

[12] S. McDonald, D. Saslow, and M. H. Alciati, "Performance and reporting of clinical breast examination: a review of the literature.," CA: a cancer journal for clinicians, vol. 54, no. 6, pp. 345-61, 2004

[13] W. H. Goodson, "Clinical Breast Examination," Topics in Primary Care Medicine, vol. 164, no. 4, pp. 355-358, 1996.

[14] N. Wang, G. J. Gerling, R. M. Childress, and M. L. Martin, "Quantifying palpation techniques in relation to performance in a clinical prostate exam.," IEEE transactions on information technology in biomedicine: a publication of the IEEE Engineering in Medicine and Biology Society, vol. 14, no. 4, pp. 1088-97, Jul. 2010.

[15] K. J. Saunders, C. A. Pilgrim, and H. S. Pennypacker, "Increased proficiency of search in breast self-examination.," Cancer, vol. 58, no. 11, pp. 2531-7, Dec. 1986.

[16] M. K. Goldstein and H. S. Pennypacker, "Improved Detection of Human Breast Lesions Fo 110 wing Experim en tal Training," Breast, pp. 408-414, 1980.

[17] M. E. Murali and K. Crabtree, "Comparison of two breast selfexamination palpation techniques.," Cancer nursing, vol. 15, no. 4, pp. 276-82, Aug. 1992.

[18] B. A. Hungerford, W. L. Gilleard, M. Moran, and C. Emmerson, "Evaluation of the ability of physical therapists to palpate intrapelvic motion with the stork test on the support side Research Report Evaluation of the Ability of Physical Therapists to Palpate Intrapelvic Motion With the Stork Test on the Support Side," Physical Therapy, vol. 87, no. 7, pp. 879-887, 2007.

[19] E. Karadogan, R. L. W. Ii, J. N. Howell, and R. R. Conatser, “A Stiffness Discrimination Experiment Including Analysis of Palpation Forces and Velocities," SImulation in Helathcare: journal of the society for SImulation in Healthcare, vol. 5, no. 4, pp. 279-88, 2010.

[20] H. T. Krouskop TA, Wheeler TM, Kallel F, Garra BS, "Elastic moduli of breast and prostate tissue under compression," Ultrasonic imaging, vol. 20, pp. 260-274, 1998.

[21] K. Sangpradit, H. Liu, L. D. Seneviratne, and K. Althoefer, "Tissue Identification using Inverse Finite Element Analysis of Rolling Indentation," in IEEE International conference on Robotics and Automation ICRA, 2009, pp. 1250-1255.

[22] M. Li, J. Konstantinova, V. Aminzadeh, T. Nanayakkara, L. D. Seneviratne, P. Dasgupta, and K. Althoefer, "Real-time Visual Stiffness Feedback for Soft Tissue Palpation in a Telemanipulation Environment," in Hamlyn Simposium 2013, 2013. 